R. J. Cohen and W. T. Sullivan, III, eds.

\title{
Chelmos (Aroania): a New European Telescope Site for the 2.3-m Telescope of the National Observatory of Athens
}

\author{
D. Sinachopoulos, F. Maragoudaki ${ }^{1}$, P. Hantzios, E. Kontizas
}

Astronomical Institute, National Observatory of Athens, PO Box 20048, GR-118 10 Athens, Greece

R. Korakitis

Dionysos Satellite Observatory, National Technical University of Athens, GR-157 80

\begin{abstract}
The National Observatory of Athens (N.O.A.) will install a new, advanced technology 2.3-m telescope in Greece. A favoured location for the installation of the new telescope is the top of Mount Chelmos. We present preliminary results concerning the conditions at this site. It is found that the site has a quite dark sky, a good percentage of clear nights (about $60 \%$ ) and very good seeing conditions.
\end{abstract}

\section{Introduction}

The National Observatory of Athens (N.O.A.) will install a new advanced technology 2.3-m telescope in Greece. The telescope will have Ritchey-Chretien optics with a field of view of 1.04 degrees. Its construction was awarded to Carl Zeiss Jena GmbH.

The National Observatory intends to install it at the top of Mount Chelmos (Aroania), at approximate coordinates longitude $22^{\circ} 12^{\prime}$ East and latitude $37^{\circ} 59^{\prime}$ North. The site is near the town of Kalavryta in northern Peloponnese, at an altitude of $2340 \mathrm{~m}$.

In satellite images (Fig.1, adapted from Cinzano et al. 2000), the site appears near the centre of one of the darkest spots of Southern Europe. We present below first results of our site tests (weather and seeing) and analysis of weather conditions based on satellite images.

\section{Weather Conditions at the Chelmos Site}

NOAA satellite weather maps (in visual and infrared) were studied in the period May 1998 to April 1999. The distribution of clear, partially clear and cloudy days is shown in Figure 2. The numbers inside the columns of the histogram indicate the actual number of days studied.

\footnotetext{
${ }^{1}$ Section of Astrophysics, Astronomy \& Mechanics, Dept. of Physics, University of Athens, GR-157 84
} 


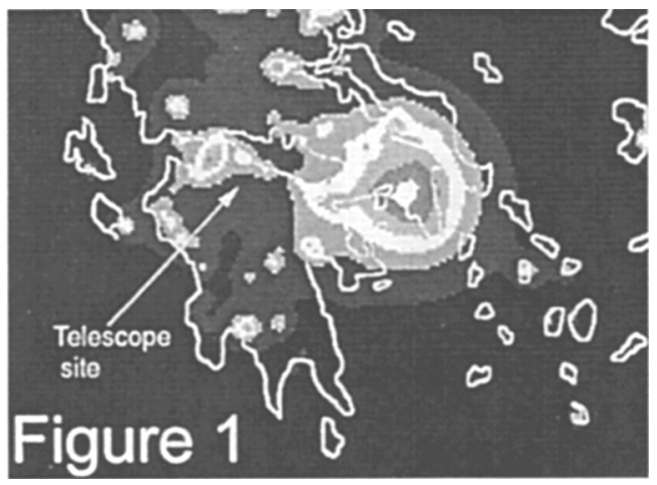

Figure 1. Location of Chelmos site in satellite image from Cinzano et al. 2000 .

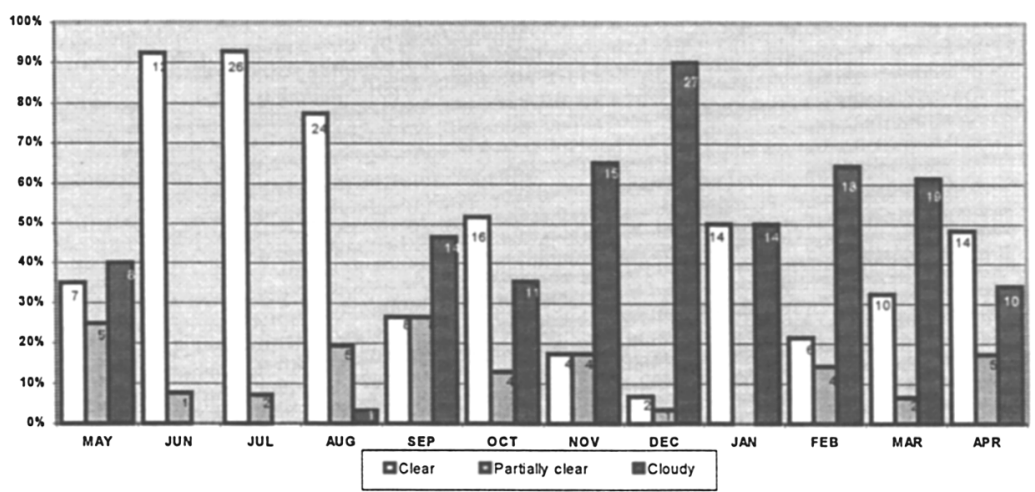

Figure 2. Chelmos weather statistics based on observations with NOAA satellites.

It is worth noting that the top of Chelmos mountain lies often above the clouds. This means that several of the cloudy days indicated in Fig. 2 are in fact clear ones at the telescope site. This is also evident after a comparison with Fig. 3, which presents the distribution of clear and cloudy days at Chelmos based on visual observations for the period December 1998 to April 1999. These observations were made at the parking lot of the nearby Winter Sports Center at an altitude of $1650 \mathrm{~m}$, which is practically always below the cloud layer. From these data we conclude that the total amount of clear nights at Chelmos is about the same as that at La Silla (Schwarz and Melnick 1993).

\section{Seeing Conditions at the Chelmos Site}

Seeing measurements were taken using the stellar trace technique (Birkle et al., 1976). A ST8 CCD camera $(1530 \times 1020$ pixels, pixel size $9 \mu \mathrm{m})$ was used for the 


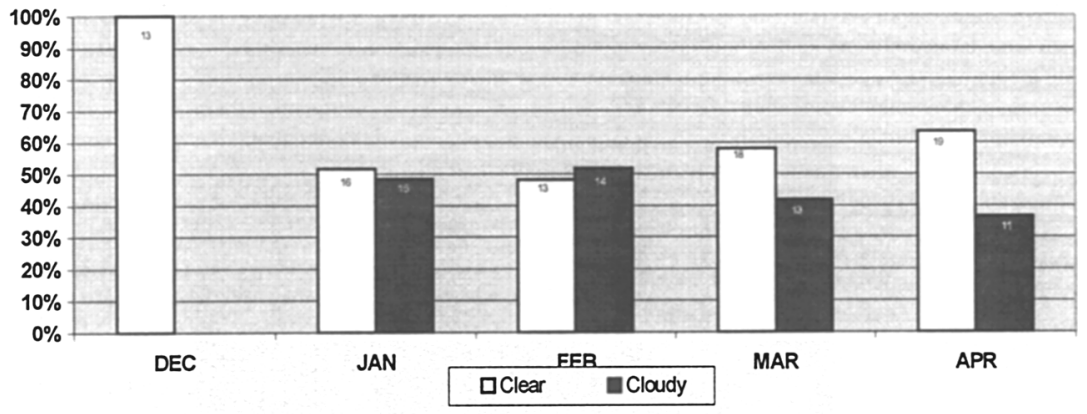

Figure 3. Chelmos weather statistics based on visual observations.

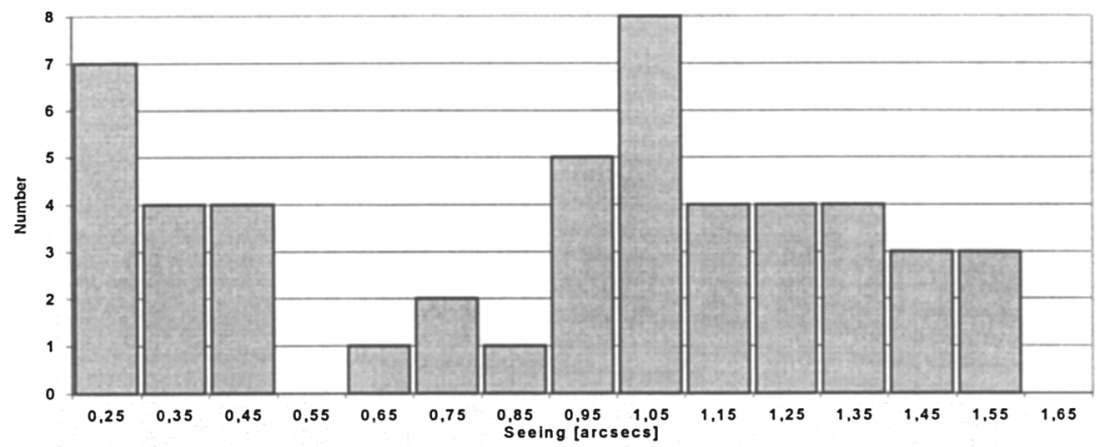

Figure 4. First seeing measurements at the Chelmos site.

observations. The preliminary results are presented in Fig. 4, which shows the amount of seeing as it is estimated from several observations during ten winter nights. These data indicate very promising seeing conditions, with a mean value around 1 arcsec and, very often, as small as 0.3 arcsec.

Acknowledgments. We express our thanks to the General Secretariat for Research and Technology for financing the new telescope project. We also thank Dr. P. Elias of the Institute for Ionospheric and Space Research of N.O.A., for his assistance in the reduction of NOAA images.

\section{References}

Birkle, K., Elsasser, H., Neckel, Th., Schnur, G. \& Schwarze, B. 1976, A\&A, 46, 397

Cinzano, P., Falchi, F., Elvidge, C.D., Baugh, K.E. 2000, MNRAS, 318, 641-657

Schwarz, H.E. \& Melnick, J. 1993, "The ESO Users Manual", 20 